\title{
Theory of Acoustic Metamaterials and Metamaterial Beams: An Overview
}

\author{
Livia Cveticanin, Gyula Mester \\ Óbuda University, Doctoral School of Safety and Security Sciences \\ Népszínház utca 8, H-1081 Budapest, Hungary \\ cpinter.livia@bgk.uni-obuda.hu,gmester@inf.u-szeged.hu
}

\begin{abstract}
This paper presents a theoretical examination of acoustic metamaterials and their application in vibration absorption. Acoustic metamaterials are concerned as analogy to electromagnetic metamaterials which are suitable for refraction and decline of electromagnetic waves at certain frequencies. Due to the analogy with these materials, the acoustic metamaterials is required to have negative effective (dynamic) mass to enable vibration elimination at the certain frequency. The concept of negative effective mass is explained based on the motion of an externally excited mass-in-mass system where the vibration elimination at the certain frequency is due to the mass-spring unit. Using these vibration absorber units, the acoustic metamaterial beams are made. Depending on the way how the units are attached to the beam, the structure may absorb waves in onedirection (for example, longitudinal waves) or waves in two directions (such as, for instance, transversal and longitudinal waves). Moreover, according to the frequency properties of the absorber units the acoustic metamaterial beams may give one, two or multi-frequency gaps. This work provides an overview of the mathematical models of acoustic metamaterial beams and also contains some suggestions for future work.
\end{abstract}

Keywords: elastic metamaterial; acoustic metamaterial beam; spring-mass systems; negative effective mass

\section{Introduction}

One of the requirements of environmental and occupant health protection is noise reduction and elimination of sources of sound pollution. Various methods are developed to damp and reduce the acoustic influence on the health of a population. One of the most often applied methods is based on the use of materials for acoustic isolation which absorb the acoustic energy. It is known that acoustic waves which come to a surface generate waves in the surface itself and these waves transmit sound power through the surface to the other side [1]. The fraction of the sound power that is transmitted to the other side is known as the transmission coefficient $T_{c}$ and the isolation effectiveness is expressed through the transmission loss $T L$ which is defined as: 


$$
T L=10 \log _{10}\left(\frac{1}{T_{c}}\right)
$$

where $\mathrm{TL}$ is in $\mathrm{dB}$. The frequencies which are of interest to be absorbed are in the low range of human hearing, approximately $100 \mathrm{~Hz}$ to $1 \mathrm{kHz}$, as for these values the irritation to health occurs. Usually, the acoustic wave transmission loss is controlled by the so-called 'mass law': the isolation mechanism is the inertia provided by the mass of the isolating partition. The transmission loss for normally incident wave propagation through a homogenous solid is estimated as:

$T L=10 \log _{10}\left[1+\left(\frac{\pi \rho h f}{c \rho_{a}}\right)^{2}\right]$

where $\rho$ is the density and $h$ is the thickness of the solid, $f$ is the frequency, $\rho_{a}$ is the density of air and $c$ is the speed of sound in the air. In [1] it is stated that at a given frequency the level of sound transmitted through a partition will be reduced by 5-6 dB for every doubling of the mass of the partition.

To improve the reduction of the acoustic effect in buildings, machinery, ships and other applications instead of acoustic isolators the novel acoustic absorbers are suggested. Their working mechanism is based on the concept of conventional vibration absorbers. As it is well known, the conventional vibration absorber consists of a lumped mass $\mathrm{m}_{2}$ attached with a linear spring $k_{2}$ to the mechanical system with mass $m_{1}$ (see Fig. 1). If the excitation force acts on the mass $m_{1}$ differential equations of motion are:

$m_{1} \ddot{u}_{1}+k_{2}\left(u_{1}-u_{2}\right)=F_{0} \exp (i \omega t)$

$m_{2} \ddot{u}_{2}+k_{2}\left(u_{2}-u_{1}\right)=0$

Solutions of (3) and (4) have the form:

$$
u_{1}=a_{1} \exp (i \omega t), \quad u_{2}=a_{2} \exp (i \omega t)
$$

where:

$a_{1}=\frac{F_{0}\left(k_{2}-m_{2} \omega^{2}\right)}{\left(k_{2}-m_{1} \omega^{2}\right)\left(k_{2}-m_{2} \omega^{2}\right)-k_{2}^{2}}$

$a_{2}=\frac{F_{0} k_{2}}{\left(k_{2}-m_{1} \omega^{2}\right)\left(k_{2}-m_{2} \omega^{2}\right)-k_{2}^{2}}$

and $i=\sqrt{-1}$ is the imaginary unit. For this model only one local resonance frequency exists. The vibration absorber uses the 1:1 external resonance between the forcing frequency on the main system $\omega$ and the local resonance frequency of the absorber $\omega_{2}=\sqrt{k_{2} / m_{2}}$ to transform the vibration energy to the absorber and stop the main system motion $\left(u_{1}=0\right)$. Based on this conception an idea of a new material, which would be the acoustic absorber is developed. Moreover, motivated by the mathematical analogy between acoustic and electromagnetic waves the investigation were directed toward so-called metamaterials which exhibit exceptional properties not observed in nature or in the constituent materials [2]. 


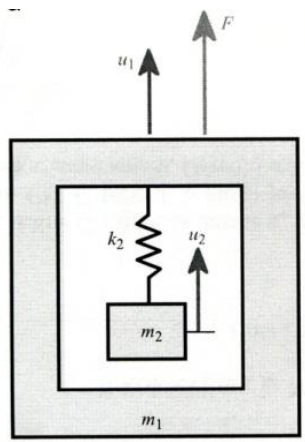

Figure 1

Mass-in-mass model [3]

Acoustic (elastic) metamaterials have to be the counterpart to electromagnetic metamaterials. The main property of electromagnetic metamaterials is that they have negative permittivity and magnetic permeability which result in a negative refractive index [4-7]. According to analogy it has to be asked the acoustic metamaterial to be with negative mass density and negative modulus. The negative effective mass/mass density is not the physical property of the material but is the result of inaccurate modeling of acoustic metamaterials.

\section{Effective Mass Density for Mass-in-Mass System}

Let us consider the mass-in-mass model (Fig.1) as a subunit of a metamaterial which is suggested to be identified with a single mass with effective mass $m_{\text {eff }}$ whose motion is the same as that of $m_{1}$. The effective mass is defined by treating this two-degree-of-freedom system as a one-degree-of-freedom one by assuming the internal absorber being unknown to the observer. In other words, the identity of the internal mass $m_{2}$ would be ignored and its effect would be absorbed by the introduction of an effective mass $m_{\text {eff }}$ as shown in Fig. 2.

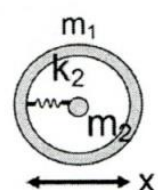

$F(t)$

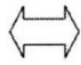<smiles>CCC</smiles>

Figure 2

If the motion of the mass $m_{1}$ is $u_{1}$, the effective mass has also the motion $u_{1}$. Linear momentums for the both models given in Fig. 2 have to be equal, i.e.: 
$m_{\text {eff }} \frac{d u_{1}}{d t}=m_{1} \frac{d u_{1}}{d t}+m_{2} \frac{d u_{2}}{d t}$

Substituting the assumed solution (5) into (7) it is:

$m_{\text {eff }} a_{1}=m_{1} a_{1}+m_{2} a_{2}$

Motion of the mass $m_{2}$ is given with the equation (4). Substituting the assumed solutions (5) we have

$-m_{2} \omega^{2} a_{2}+k_{2}\left(a_{2}-a_{1}\right)=0$

After some modification equations (8) and (9) yield the effective mass [8-10]:

$m_{\text {eff }}=m_{1}+m_{2} \frac{k_{2}}{k_{2}-m_{2} \omega^{2}}$

which is for: $\omega_{2}=\sqrt{k_{2} / m_{2}}$

$m_{e f f}=m_{1}+m_{2} \frac{\omega_{2}^{2}}{\omega_{2}^{2}-\omega^{2}}$

Analyzing the relation (11) it is obvious that the effective mass depends on the ration between the excitation frequency $\omega$ and natural frequency of the system $\omega_{2}$ :

$m_{e f f}=m_{1}+m_{2} \frac{1}{1-\frac{\omega^{2}}{\omega_{2}^{2}}}$

For the system three modes of motion are evident: 1) acoustic mode when $\omega<\omega_{2}$, 2) resonant mode when $\omega=\omega_{2}$ and 3) optic mode when $\omega>\omega_{2}$. For the acoustic mode the effective mass is positive. In the resonant mode the effective mass is theoretically infinite. In the optical mode the effective mass is negative for:

$m_{1}+m_{2} \frac{1}{1-\frac{\omega^{2}}{\omega_{2}^{2}}}<0$.

Otherwise, it is positive. In Fig. 3, according to (12) the effective mass - frequency diagram is plotted

Differentiating the relation (7) we have:

$\left(m_{\text {eff }}-m_{1}\right) \ddot{u}_{1}=m_{2} \ddot{u}_{2}$

Substituting (14) into (4) we obtain:

$k_{2}\left(u_{2}-u_{1}\right)=-\left(m_{e f f}-m_{1}\right) \ddot{u}_{1}$

Equation (3) and (15) give:

$-m_{e f f} \ddot{u}_{1}=F_{0} \exp (i \omega t)$

The effective mass is the ratio between the excitation force and acceleration of the mass $m_{1}$ :

$m_{e f f}=\frac{F}{\ddot{u}_{1}}=\frac{F_{0}}{-\omega^{2} a_{1}}=-\frac{F}{\omega^{2} u_{1}}$ 


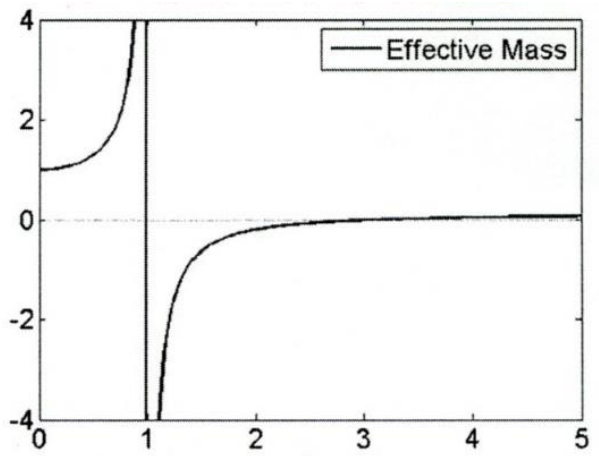

Figure 3

Dimensionless effective mass $m_{e f f} / m_{1}$ as a function of $\omega / \omega_{2}$ [10]

According to Fig. 3 it is obvious that for $\omega_{2}=\omega$ the effective mass tends to infinity. For that value the motion of mass $m_{1}$ is zero and the inertial force of the mass $m_{2}$ is equal to the excitation force: $F(t)=m_{2} \ddot{u}_{2}$. So, the external force is eliminated with the inertia force $-m_{2} \ddot{u}_{2}$ through the spring $k_{2}$. This is the concept of vibration absorbers.

Finally, the following is concluded:

In the acoustic mode, when $\omega<\omega_{2}$, the effective mass $m_{\text {eff }}$ is positive and the motions $u_{1}$ and $u_{2}$ are in phase. For the optical mode, when $\omega>\omega_{2}$, the effective mass $m_{\text {eff }}$ may be positive or negative, while the displacements $u_{1}$ and $u_{2}$ are $180^{\circ}$ out of phase. Then, the absorber works efficiently in the optical mode against the external acting on the mass $m_{1}$. The excitation is absorbed with the inertial force.

\section{Mechanical Structure with Negative Effective Mass}

Mechanical structures are designed by incorporating of the previously mentioned mechanical subunits in a natural material with the aim to resonate during mechanical wave propagation in it. For the local mechanical resonance the designed structures have negative effective masses. The negative mass behavior is generated by oscillating of resonant structures within the material with $180^{\circ}$ out of phase to the acoustic waves which apply to surface. It causes existence of frequency bands where wave propagation is theoretically impossible. These bands are usually called band gaps. The aim of the designed structure is to overcome the limitations of the mass law for solids by creating engineered materials with useful acoustic band gaps, and the key to the generation of these band gaps is an inhomogeneous structure. Huang et al. [10] composed a one-dimensional lattice which contains mass-in-mass lattices (Fig. 4). The model is based on those with negative mass as explained in [7] and [8]. Equations of motion for the unit cell are: 


$$
\begin{aligned}
& m_{1}^{(j)} \ddot{u}_{1}^{(j)}+k_{2}\left(u_{1}^{(j)}-u_{2}^{(j)}\right)+k_{1}\left(2 u_{1}^{(j)}-u_{1}^{(j-1)}-u_{1}^{(j+1)}\right)=0 \\
& m_{2}^{(j)} \ddot{u}_{2}^{(j)}+k_{2}\left(u_{2}^{(j)}-u_{1}^{(j)}\right)=0
\end{aligned}
$$

where $k_{1}$ is the rigidity of connection.

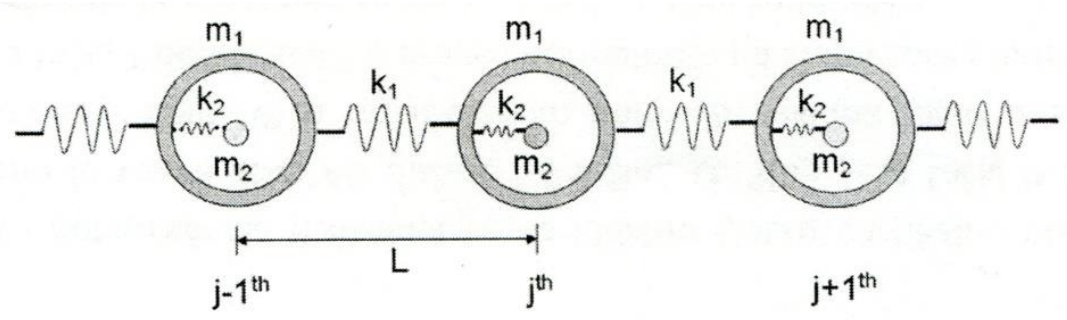

Figure 4

Model of subunits connected in lattice [11]

The harmonic wave solution for (18) is assumed in the form:

$u_{1}^{(j)}=U_{1} \exp (i \beta x-i \omega t)$

$u_{2}^{(j)}=U_{2} \exp (i \beta x-i \omega t)$

$u_{1}^{(j+1)}=U_{1} \exp (i \beta x-i \omega t) \exp (i \beta L)$

$u_{1}^{(j-1)}=U_{1} \exp (i \beta x-i \omega t) \exp (-i \beta L)$

Substituting (19) into (18) two homogenous equations for $U_{1}$ and $U_{2}$ follow which give the dispersion equation:

$m_{1} m_{2} \omega^{4}-\left[\left(m_{1}+m_{2}\right) k_{2}+2 m_{2} k_{1}(1-\cos (\beta L))\right] \omega^{2}+2 k_{1} k_{2}(1-\cos (\beta L))=0$

In Fig. 5, both branches of the band structure (20) which correspond to the optical mode, when $\omega>\omega_{0}$, and to the acoustic mode, when $\omega<\omega_{0}$, are plotted. The parameter values are $m_{2} / m_{1}=9, \quad k_{2} / k_{1}=0.1$ and $\omega_{0}=\sqrt{k_{2} / m_{2}}=149.07 \mathrm{~s}^{-1}$. The frequency distribution is given as a function of the wave number $\beta L$.

The displacements in (19) are functions of $\exp (i \beta x)$. For the case when the wave number has a complex form:

$\beta L=\gamma+i \alpha$

it is

$u \propto \exp \left(\frac{i \gamma x}{L}\right) \exp \left(-\frac{\alpha x}{L}\right)$. 


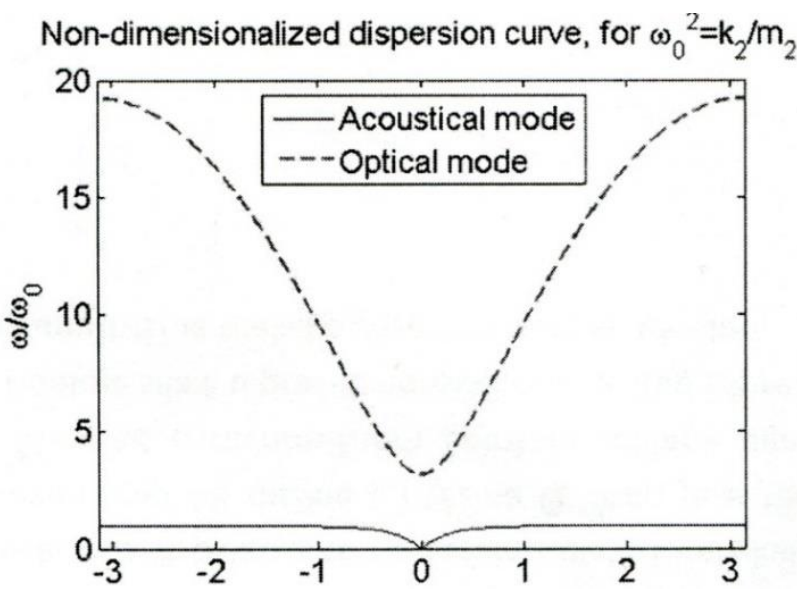

Figure 5

Nondimensional dispersion curve for the mass-in-mass lattice model [10]

The amplitude of the displacement decays as the exponential function $\exp \left(-\frac{\alpha x}{L}\right)$ if the attenuation factor $\gamma$ is positive. It is of interest to analyze the case when wave frequency approaches the local resonance frequency $\omega_{0}$ and the attenuation factor $\gamma$ theoretically becomes unbounded.

If the lattice system is reduced to a homogenous mono-atom lattice system in which only effective masses $m_{\text {eff }}$ are connected by springs with rigidity $k_{1}$, the homogenous lattice system has the dispersion equation:

$\omega^{2}=\frac{2 k_{1}}{m_{e f f}}(1-\cos (\beta L))$

The suggested model is equivalent to the original mass-in-mass system if their dispersion equations (20) and (23) are identical. Substituting (23) into (20) the effective mass is obtained:

$$
m_{e f f}=m_{s t}+\frac{m_{2}\left(\frac{\omega}{\omega_{0}}\right)^{2}}{1-\left(\frac{\omega}{\omega_{0}}\right)^{2}}
$$

where $m_{s t}=m_{1}+m_{2}$. Comparing (24) with the result (12) it is seen that they are identical and that the diagram shown in Fig. 3, is also valid here. Let us extend the discussion of the mentioned diagram. Especially the vibrations out of resonant region are considered. Thus, for frequencies far below the local resonance, the internal masses oscillate in phase with the solid in which they are embedded. But as the excitation frequencies passes through the resonance and the effective mass becomes negative near the local resonance frequency $\omega_{0}$, the phase angle of the response changes by close to $180^{\circ}$. That means that near this frequency the acceleration of the resonant structures within the metacomposite will have a component whose direction is opposite to that of the force of pressure applied to the surface of the metacomposite. 
However, the most important rule is the resonant one. Analyzing relations (23) and (24) it is obvious that the effective mass is negative only when $(1-\cos (\beta L))$ is negative and when $k_{1}$ and $\omega^{2}$ are positive. It means that the dimensionless wavenumber $\beta L$ has to be complex. Thus, frequencies corresponding to the negative mass are in the stopping band. In other words, a negative effective mass in the equivalent mass-spring lattice system yields spatial attenuation in wave amplitude. For a material with a negative mass density the speed of sound is imaginary, and therefore, only evanescent waves, which decay exponentially from the surface, can exist.

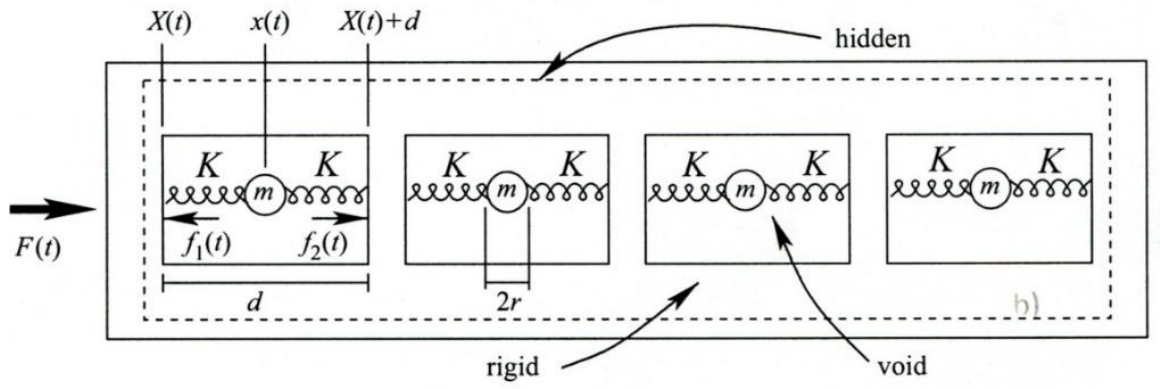

Figure 6

A one-dimensional material where the mass depends on the frequency $\omega$ and can be negative [8].

Based on this theoretical consideration, Milton and Willis [8], developed a barlike acoustic metamaterial with heterogeneous material properties which are valid for waves of wavelengths much longer than the sizes of subunits. Milton and Willis [8] concluded that solids containing many identical small resonators [8] exhibit band gap behavior near their resonance frequency, even though the size and spacing of the resonators were over a 100 times smaller than the wavelength at the band gap frequency. Furthermore, lack of order of periodicity in the arrangement of the resonators did not materially affect the results, and the band gap frequencies could be tuned through changes to the resonators natural frequencies. These locally resonant sonic materials (LRSM), shown in Fig. 4, are considered to be a type of acoustic absorbers [11-13]. The model of the structure is one-dimensional [8]. From a bar, made of rigid material, cylindrical cavities of length $d$ have been carved out. Each cavity is modeled as a sphere of mass $m$ and radius $r$. The sphere is attached to the ends of the cavity with two possibly viscoelastic springs each having the same complex spring constant $K$. It is assumed that everything is varied harmonically with time with frequency $\omega$. The model is plotted in Fig. 6.

In Fig. 7 the fabricated lattice beam is shown. The model is suitable to describe the propagation of the dispersive wave in the lattice. 
(a)

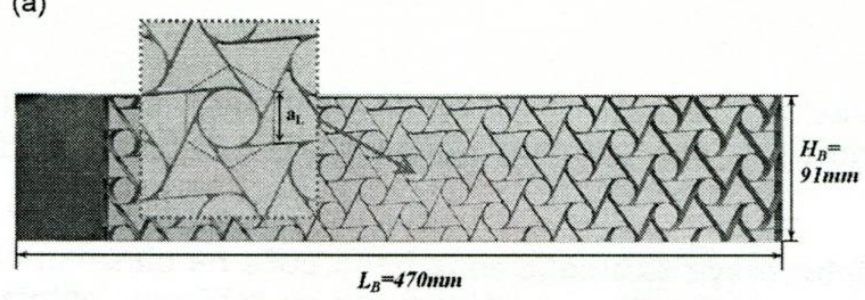

(b)

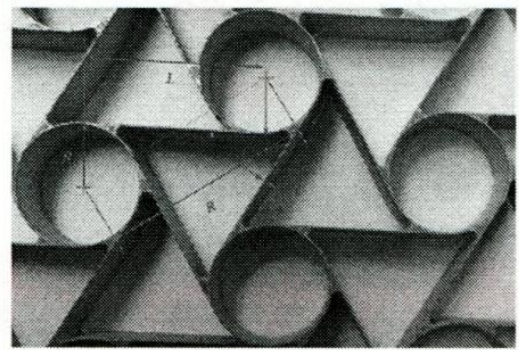

Figure 7

a) The fabricated chiral lattice beam and its zoomed unit cell and b) the topology of the hexagonal chiral lattice [14]

This structure is non-homogenous. If it is required that the metamaterial behave like a homogeneous material described by its averaged material properties, its subunits must be much smaller than the shortest wavelength of waves propagating in it. The averaging may result in the existence of a useful but mysterious phononic stop-band that allows no waves within that frequencies range to propagate forward, and most current designs of acoustic metamaterials are based on the stop-band effect [10]. For manufacturing such metamaterials with tiny subunits in order to have stop-bands, expensive manufacturing techniques are required including micro and nano-manufacturing technologies.

\section{Design of Metamaterial Beams for Broadband Absorption and Isolation}

To eliminate the lack of the previously mentioned beam-absorbers made of LRSM and of the chiral lattice beam, acoustic absorber based on a metamaterial beam is developed. The metamterial beam consists of a uniform isotropic beam with many small spring-mass-damper subsystems integrated at separated locations along the beam to act as vibration absorbers (Fig. 8). The spring-mass-damper subsystems create a stop-band in which no elastic waves in this frequency range can propagate forward. The concepts of negative effective mass and stiffness is applied for metamaterial design. 


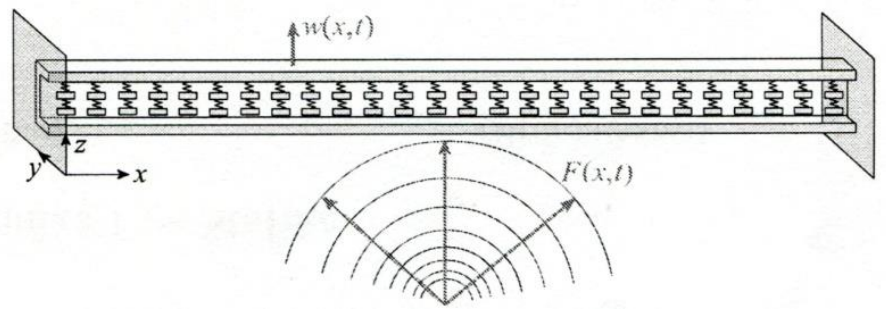

Figure 8

Model of a metamaterial beam for vibration absorption [2].

The proposed metamaterial beam is based on the concept of conventional mechanical vibration absorbers. It uses the incoming elastic wave in the beam to resonate the integrated spring-mass-damper absorbers to vibrate in their optical mode at frequencies close to but above their local resonance frequencies to create shear forces and bending moments to straighten the beam and stop the wave propagation. Metamaterials usually designed as metacomposites have unusual response to elastic waves.

\subsection{Metamaterial Beams for Broadband Absorption of Longitudinal Elastic Waves}

In Fig. 9, a metamaterial beam for acoustic absorption is presented. In the longitudinal beam spring-mass subsystems are integrated. The beam has to absorb low and high frequency elastic waves [3]. The absorption of the longitudinal elastic waves is based on the negative mass effect. The concept of the negative effective mass and vibration absorbers with two-degrees-of-freedom, mass-inmass system, forced with harmonic excitation is demonstrated in Section 2. It is shown that the negative effective mass density gives the stop band when the incoming wave frequency is slightly higher than the local frequency.

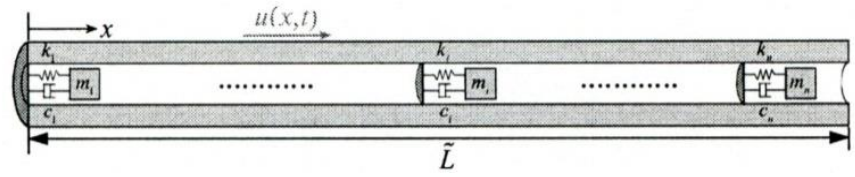

Figure 9

Wave propagation in a metamaterial beam [12].

Using the concept of vibration absorbers, the unit cell model is shown in Fig. 10. The equations for a unit cell of an infinite metamaterial beam can be derived using the extended Hamiltonian principle.

$\int_{0}^{L}\left(\delta T-\delta U+\delta W_{n c}\right)=0$

where $T$ is kinetic energy, $U$ is the elastic energy and $W_{n c}$ is the work of the nonconservative forces. 


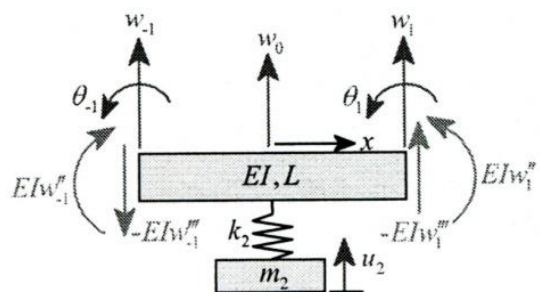

Figure 10

The unit cell model

For the unit cell the kinetic energy is:

$\delta T=-\int_{-\frac{L}{2}}^{\frac{L}{2}} \rho A d x \frac{\partial^{2} w}{\partial t^{2}} \delta w-m_{2} \frac{\partial^{2} u_{2}}{\partial t^{2}} \delta u_{2}$

the elastic energy is:

$$
\begin{aligned}
& \delta U=k_{2}\left(u_{2}-w_{0}\right) \delta\left(u_{2}-w_{0}\right)+\int_{-\frac{L}{2}}^{\frac{L}{2}} E I \frac{\partial^{4} w}{\partial x^{4}} d x \delta w \\
& +E I\left(\frac{\partial^{2} w_{1}}{\partial x^{2}}\right) \delta\left(\frac{\partial w_{1}}{\partial x}\right)-E I\left(\frac{\partial^{2} w_{-1}}{\partial x^{2}}\right) \delta\left(\frac{\partial w_{-1}}{\partial x}\right) \\
& -E I\left(\frac{\partial^{3} w_{1}}{\partial x^{3}}\right) \delta w_{1}+E I\left(\frac{\partial^{3} w_{-1}}{\partial x^{3}}\right) \delta w_{-1}+E I\left(\frac{\partial^{3} w_{0+}}{\partial x^{3}}\right) \delta w_{0}+E I\left(\frac{\partial^{3} w_{0-}}{\partial x^{3}}\right) \delta w_{0}
\end{aligned}
$$

and the work of the nonconservative forces is:

$$
\begin{aligned}
& \delta W_{n c}=E I\left(\frac{\partial^{2} w_{1}}{\partial x^{2}}\right) \delta\left(\frac{\partial w_{1}}{\partial x}\right)-E I\left(\frac{\partial^{2} w_{-1}}{\partial x^{2}}\right) \delta\left(\frac{\partial w_{-1}}{\partial x}\right) \\
& -E I\left(\frac{\partial^{3} w_{1}}{\partial x^{3}}\right) \delta w_{1}+E I\left(\frac{\partial^{3} w_{-1}}{\partial x^{3}}\right) \delta w_{-1}
\end{aligned}
$$

where $\left(\frac{\partial w}{\partial x}\right)=\theta$. It is $E I\left(\frac{\partial^{3} w_{0+}}{\partial x^{3}}\right) \neq E I\left(\frac{\partial^{3} w_{0-}}{\partial x^{3}}\right)$ because the vibration absorber creates a concentrated shear force at $x=0$. Substituting (26) - (28) into (25) and separating the terms with $\delta w$ and $\delta u_{2}$ the following equations are obtained:

$0=-\rho A \frac{\partial^{2} w}{\partial t^{2}}-E I \frac{\partial^{4} w}{\partial x^{4}}+\left[k_{2}\left(u_{2}-w_{0}\right)+E I\left(\frac{\partial^{3} w_{0+}}{\partial x^{3}}\right)+E I\left(\frac{\partial^{3} w_{0-}}{\partial x^{3}}\right)\right] \delta(x)$

$m_{2} \frac{\partial^{2} u_{2}}{\partial t^{2}}+k_{2}\left(u_{2}-w_{0}\right)=0$

where $\delta(x)$ is the Dirac function. Due to periodicity along $x$ direction, flexural wave propagation through the infinite periodic beam can be expressed in a harmonic form:

$w(x, t)=W \exp (i \beta x-i \omega t), \quad w_{0}(x, t)=W_{0} \exp (-i \omega t)$ 
$u_{2}(x, t)=U_{2} \exp (-i \omega t)$

where $i=\sqrt{-1}$ is the imaginary unit, $\beta$ is the wave number and $\omega$ is the vibration frequency.

If the metamaterial is treated as a homogenized uniform beam the integration over the whole length gives:

$0=k_{2}\left(u_{2}-w_{0}\right)-\int_{-\frac{L}{2}}^{\frac{L}{2}} \rho A \frac{\partial^{2} w}{\partial t^{2}} d x-E I\left(\frac{\partial^{3} w_{1}}{\partial x^{3}}\right)+E I\left(\frac{\partial^{3} w_{-1}}{\partial x^{3}}\right)$

Using the harmonic wave solution and the assumption that the beam segment is a lumped mass $\widetilde{m}$ and a spring $\tilde{k}$ system, we have:

$\tilde{m} \frac{\partial^{2} w_{0}}{\partial t^{2}}+\tilde{k} w_{0}+k_{2}\left(u_{2}-w_{0}\right)=0$

where:

$\tilde{m}=-\frac{2 \rho A \sin \left(\frac{\beta L}{2}\right)}{\beta}, \quad \tilde{k}=-2 E I \beta^{3} \sin \left(\frac{\beta L}{2}\right)$

The values $\tilde{m}$ and $\tilde{k}$ depend on the wavenumber $\beta$. Substituting (30) into (29) and (32), and equating the determinant of corresponding parameters with zero, we have:

$\left[\begin{array}{cc}-\tilde{m} \omega^{2}+\tilde{k}+k_{2} & -k_{2} \\ -k_{2} & -m_{2} \omega^{2}+k_{2}\end{array}\right]=0$

The dispersion equation follows as:

$\left(-\tilde{m} \omega^{2}+\tilde{k}+k_{2}\right)\left(-m_{2} \omega^{2}+k_{2}\right)-k_{2}^{2}=0$

To each specific value of $\omega$ the positive value of $\beta$ has to be determined. If no positive values for $\beta$ exist, the value of $\omega$ is the stop band. Namely, if $\beta=i \alpha$ and $\alpha>0$, and:

$w(x, t)=W \exp (-\alpha x) \exp (-i \omega t)$

there is an evanescent non-propagating wave and the stop band exists.

\subsection{Metamaterial Beams with Two Spring-Mass Systems for Broadband Absorption of Longitudinal Elastic Waves}

The metamaterial previously considered has single-mass absorbers and produces a stop band at the high-frequency side. Pai et al., [12] presented a new metamaterial beam based on multi-frequency vibration absorbers for broadband vibration absorption.

The metamaterial beam consists of a uniform isotropic beam and small two-mass spring-mass-damper subsystems at many locations along the beam to act as multifrequency vibration absorber. This type of absorber produces two stop-bands. 
The existence of stop-bands is caused by spring-mass-damper subsystems and their existence is explained according to negative effective mass and effective stiffness.

For an incoming wave with a frequency in one of the two stop-bands, the absorbers are excited to vibrate in their optical modes to create shear forces to straighten the beam and stop the wave propagation. For an incoming wave with a frequency outside of but between the two stop-bands, it can be damped by the damper with the second mass of each absorber. So, the stop-bands are connected into a wide stop-band.

To show the use of multi-frequency vibration absorbers to design broadband metamaterials Pai et al. [2] consider the three-degree-of-freedom oscillating system shown in Fig. 11. On the mass $m_{1}$, connected with the spring $k_{1}$ and damping $c_{1}$, a harmonic excitation force acts. The vibration absorber uses two lumped masses $m_{2}$ and $m_{3}$ connected with springs $k_{2}$ and $k_{3}$ and damping coefficients $c_{2}$ and $c_{3}$.

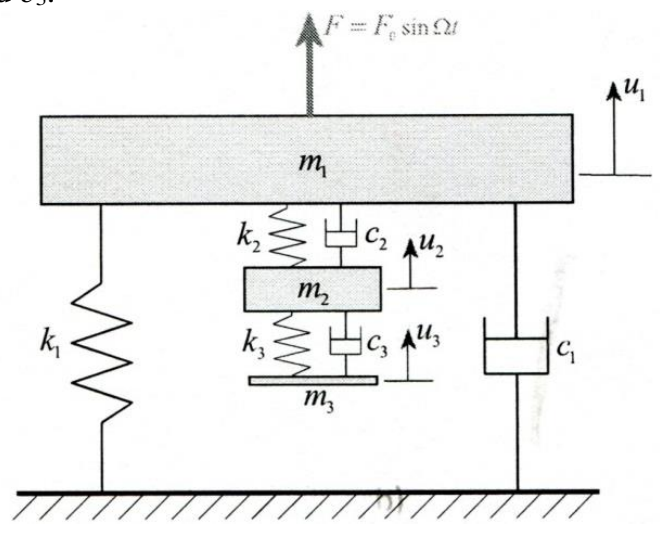

Figure 11

Three-degree-of-freedom oscillatory system [2]

We describe the motion of the system with a system of coupled differential equations

$$
\begin{aligned}
& m_{1} \ddot{u}_{1}+\left(c_{1}+c_{2}\right) \dot{u}_{1}-c_{2} \dot{u}_{2}+\left(k_{1}+k_{2}\right) u_{1}-k_{2} u_{2}=F_{0} \exp (i \omega t) \\
& m_{2} \ddot{u}_{2}+\left(c_{2}+c_{3}\right) \dot{u}_{2}-c_{2} \dot{u}_{1}-c_{3} \dot{u}_{3}+\left(k_{2}+k_{3}\right) u_{2}-k_{2} u_{1}-k_{3} u_{3}=0 \\
& m_{3} \ddot{u}_{3}+c_{3}\left(\dot{u}_{3}-\dot{u}_{2}\right)+k_{3}\left(u_{3}-u_{2}\right)=0
\end{aligned}
$$

The aim of the absorber is to make $u_{1}=0$ by adjusting one of two local natural frequencies equal to the excitation frequency $\omega$. For the undamped system for which $u_{1}=0$ equations (37) simplify to:

$m_{2} \ddot{u}_{2}+\left(k_{2}+k_{3}\right) u_{2}-k_{3} u_{3}=0$

$m_{3} \ddot{u}_{3}+k_{3}\left(u_{3}-u_{2}\right)=0$

where the natural frequencies are: 
$\omega_{1 n}, \omega_{2 n}=\omega_{2} \sqrt{\frac{\bar{m}+\bar{k}+\bar{m} \bar{k} \pm \sqrt{(\bar{m}+\bar{k}+\bar{m} \bar{k})^{2}-4 \bar{m} \bar{k}}}{2 \bar{m}}}$

where $\bar{m}=\frac{m_{3}}{m_{2}}, \bar{k}=\frac{k_{3}}{k_{2}}, \omega_{2}=\sqrt{\frac{k_{2}}{m_{2}}}$. Around these two frequencies two stop-bands exist.

The goal is to design metamaterial beam based on the vibration absorber shown in Fig. 12 that can stop propagation of elastic waves. The unit cell model is plotted in Fig. 12a. Using the relation (25), equations for a unit cell model are derived.

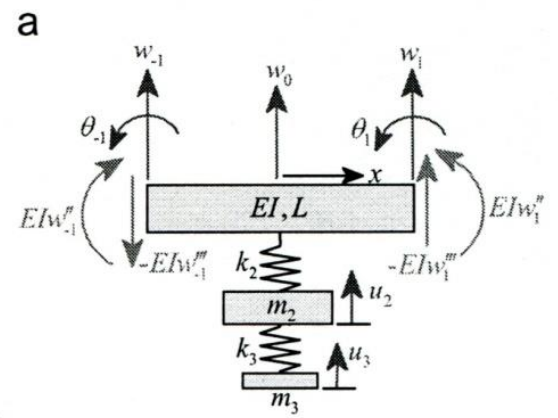

b

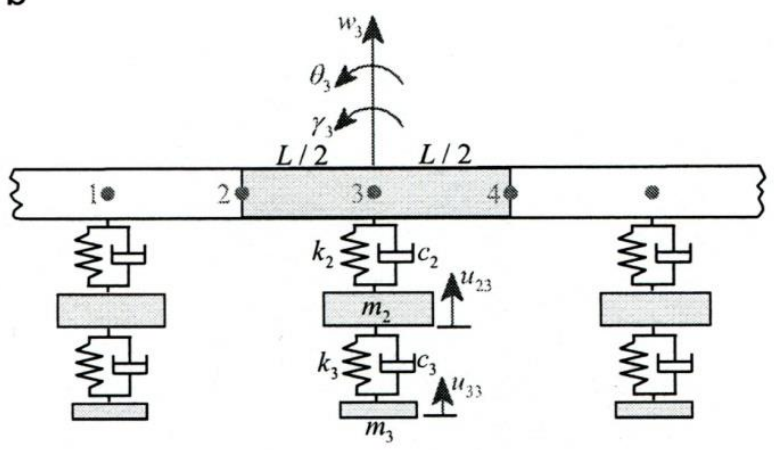

Figure 12

Model of an infinite metamaterial beam: a) a unit cell, b) an infinite beam [2]

The kinetic energy, elastic energy and the work of the non-conservative forces are:

$$
\begin{aligned}
& \delta T=-\int_{-\frac{L}{2}}^{\frac{L}{2}} \rho A d x \frac{\partial^{2} w}{\partial t^{2}} \delta w-m_{2} \frac{\partial^{2} u_{2}}{\partial t^{2}} \delta u_{2}-m_{3} \frac{\partial^{2} u_{3}}{\partial t^{2}} \delta u_{3} \\
& \delta U=k_{2}\left(u_{2}-w_{0}\right) \delta\left(u_{2}-w_{0}\right)+\int_{-\frac{L}{2}}^{\frac{L}{2}} E I \frac{\partial^{4} w}{\partial x^{4}} d x \delta w \\
& +E I\left(\frac{\partial^{2} w_{1}}{\partial x^{2}}\right) \delta\left(\frac{\partial w_{1}}{\partial x}\right)-E I\left(\frac{\partial^{2} w_{-1}}{\partial x^{2}}\right) \delta\left(\frac{\partial w_{-1}}{\partial x}\right)+k_{3}\left(u_{3}-u_{2}\right) \delta\left(u_{3}-u_{2}\right) \\
& -E I\left(\frac{\partial^{3} w_{1}}{\partial x^{3}}\right) \delta w_{1}+E I\left(\frac{\partial^{3} w_{-1}}{\partial x^{3}}\right) \delta w_{-1}+E I\left(\frac{\partial^{3} w_{0+}}{\partial x^{3}}\right) \delta w_{0}+E I\left(\frac{\partial^{3} w_{0-}}{\partial x^{3}}\right) \delta w_{0}
\end{aligned}
$$




$$
\begin{aligned}
& \delta W_{n c}=E I\left(\frac{\partial^{2} w_{1}}{\partial x^{2}}\right) \delta\left(\frac{\partial w_{1}}{\partial x}\right)-E I\left(\frac{\partial^{2} w_{-1}}{\partial x^{2}}\right) \delta\left(\frac{\partial w_{-1}}{\partial x}\right) \\
& -E I\left(\frac{\partial^{3} w_{1}}{\partial x^{3}}\right) \delta w_{1}+E I\left(\frac{\partial^{3} w_{-1}}{\partial x^{3}}\right) \delta w_{-1}
\end{aligned}
$$

where $\left(\frac{\partial w}{\partial x}\right)=\theta$. It is $E I\left(\frac{\partial^{3} w_{0+}}{\partial x^{3}}\right) \neq E I\left(\frac{\partial^{3} w_{0-}}{\partial x^{3}}\right)$ because the vibration absorber creates a concentrated shear force at $x=0$. Substituting (40) - (42) into (25) and separating the terms with $\delta w, \delta u_{2}$ and $\delta u_{3}$ the following three equations are obtained:

$0=-\rho A \frac{\partial^{2} w}{\partial t^{2}}-E I \frac{\partial^{4} w}{\partial x^{4}}+\left[k_{2}\left(u_{2}-w_{0}\right)+E I\left(\frac{\partial^{3} w_{0+}}{\partial x^{3}}\right)+E I\left(\frac{\partial^{3} w_{0-}}{\partial x^{3}}\right)\right] \delta(x)$

$m_{2} \frac{\partial^{2} u_{2}}{\partial t^{2}}+k_{2}\left(u_{2}-w_{0}\right)+k_{3}\left(u_{2}-u_{3}\right)=0$

$m_{3} \frac{\partial^{2} u_{3}}{\partial t^{2}}+k_{3}\left(u_{3}-u_{2}\right)=0$

where $\delta(x)$ is the Dirac function. Due to periodicity along $x$ direction, wave propagation through the infinite periodic beam can be expressed in a harmonic form:

$w(x, t)=W \exp (i \beta x-i \omega t), \quad w_{0}(x, t)=W_{0} \exp (-i \omega t)$,

$u_{2}(x, t)=U_{2} \exp (-i \omega t), \quad u_{3}(x, t)=U_{3} \exp (-i \omega t)$

where $i=\sqrt{-1}$ is the imaginary unit, $\beta$ is the wave number and $\omega$ is the vibration frequency. If the metamaterial is treated as a homogenized uniform beam the integration over the whole length (see Fig. 12b) gives:

$0=k_{2}\left(u_{2}-w_{0}\right)-\int_{-\frac{L}{2}}^{\frac{L}{2}} \rho A \frac{\partial^{2} w}{\partial t^{2}} d x-E I\left(\frac{\partial^{3} w_{1}}{\partial x^{3}}\right)+E I\left(\frac{\partial^{3} w_{-1}}{\partial x^{3}}\right)$

Using the harmonic wave solution and the assumption that the beam segment is a lumped with mass $\tilde{m}$ and spring rigidity $\tilde{k}$, we obtain the equation (32) with explanation (33). The values $\widetilde{m}$ and a spring $\tilde{k}$ depend on the wavenumber $\beta$. Substituting (46) into (32), (44) and (45), and separating the terms with $W_{0}, U_{2}$ and $U_{3}$ the determinant of the system is formed. The solution of the system is nontrivial if the determinant is zero, i.e.,

$$
\left[\begin{array}{ccc}
-\tilde{m} \omega^{2}+\tilde{k}+k_{2} & -k_{2} & 0 \\
-k_{2} & k_{2}+k_{3}-\omega^{2} m_{2} & -k_{3} \\
0 & -k_{3} & k_{3}-\omega^{2} m_{3}
\end{array}\right]=0
$$

Developing the determinant (48) the dispersion equation is obtained:

$$
\left(-\tilde{m} \omega^{2}+\tilde{k}+k_{2}\right)\left[\left(-\tilde{m} \omega^{2}+\tilde{k}+k_{2}\right)\left(k_{3}-\omega^{2} m_{3}\right)-k_{3}^{2}\right]-k_{2}^{2}\left(k_{3}-\omega^{2} m_{3}\right)=0
$$

For the solution of the dispersion equation in which the wave number $\beta=i \alpha$ and $\alpha>0$, the function $w$ has the form (36). It is obvious that the amplitude decreases and tends to zero, i.e., the stop-band exists. 


\subsection{Multi-Flexural Band Gaps in an Euler-Bernoulli Beam with Lateral Local Resonators Transformation of the Flexural into Longitudinal Vibrations}

In the previous text, single and double band gap metamaterials are shown, which are not suitable for the multi-frequency wave suppression. Further, in the configurations the local resonators are attached to continuum beams to generate band gaps for stopping the propagation of waves in one (longitudinal or lateral or torsional) direction. Wang et al. [15] suggested an acoustic metamaterial beam which is based on the multi-frequency vibration absorption and transformation of the flexural waves into longitudinal, i.e., the flexural vibration is attenuated into another direction in a beam. For theoretical consideration of the dynamic characteristics of the flexural wave propagation in an Euler-Bernoulli beam with lateral local resonators (LLR) the mathematical model is formed. Namely, it is of interest to suppress the flexural vibration in such a beam. The LLR structures substructures partially transform the flexural waves into longitudinal waves and block the wave propagation in another direction. In Fig. 13 a simple model of an Euler-Bernoulli beam with periodical LLR substructures in $x$ direction is plotted. One LLR consists of two lateral resonators with spring and mass constant of $k_{2}$ and $m_{2}$ and a vertical resonator with spring and mass constant $k_{1}$ and $m_{1}$ and a four link mechanism with rigid and massless trusses. The beam and the vertical resonator vibrate in $z$ direction and the lateral resonators vibrate in $x$ direction with displacement $w, u_{1}$ and $u_{2}$, respectively.

(a)

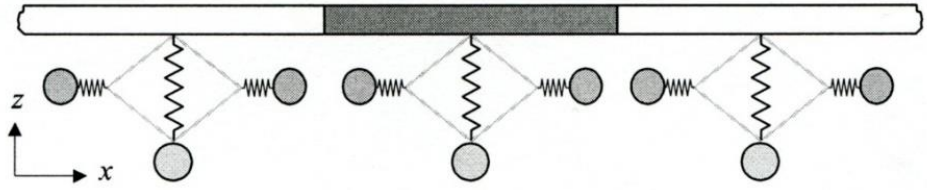

(b)

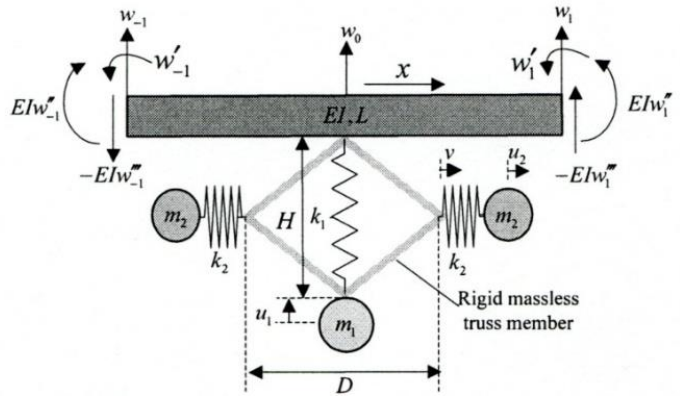

Figure 13

Construction of metamaterial beam: a) an infinite beam, b) a typical unit cell [15]

The unit cell of an infinite periodic metamaterial beam is shown in Fig. 13b. Inertial force in $z$ direction of the elementary beam is:

$\rho A d x \frac{\partial^{2} w}{\partial t^{2}}$ 
Inertial forces of masses are: $m_{1} \frac{\partial^{2} u_{1}}{\partial t^{2}}$ and $m_{2} \frac{\partial^{2} u_{2}}{\partial t^{2}}$. Then, the elementary kinetic energy is:

$\delta T=-m_{1} \frac{\partial^{2} u_{1}}{\partial t^{2}} \delta u_{1}-2 m_{2} \frac{\partial^{2} u_{2}}{\partial t^{2}} \delta u_{2}-\int_{-L / 2}^{L / 2} \rho A d x \frac{\partial^{2} w}{\partial t^{2}} \delta w$

The elastic force of the unit beam is $E I \frac{\partial^{4} w}{\partial x^{4}} d x$ and the elastic forces in springs are $k_{1}\left(u_{1}-w_{0}\right)$ and $k_{2}\left(u_{2}-v\right)$ where $w_{0}$ is the flexural displacement of the center of beam and $v$ is the displacement of the truss end connected the lateral resonators. Based on the assumption of small displacements, we have:

$v=-\frac{H}{2 D}\left(w_{0}-u_{1}\right)$

Introducing the boundary conditions for the elementary unit, the elementary elastic energy of the system is:

$\delta U=k_{1}\left(u_{1}-w_{0}\right) \delta\left(u_{1}-w_{0}\right)+\int_{-\frac{L}{2}}^{\frac{L}{2}} E I \frac{\partial^{4} w}{\partial x^{4}} d x \delta w+2 k_{2}\left(u_{2}+\frac{H}{2 D}\left(w_{0}-\right.\right.$

$\left.\left.u_{1}\right)\right) \delta\left(u_{2}+\frac{H}{2 D}\left(w_{0}-u_{1}\right)\right)+E I\left(\frac{\partial^{2} w_{1}}{\partial x^{2}}\right) \delta\left(\frac{\partial w_{1}}{\partial x}\right)-E I\left(\frac{\partial^{2} w_{-1}}{\partial x^{2}}\right) \delta\left(\frac{\partial w_{-1}}{\partial x}\right)$

$-E I\left(\frac{\partial^{3} w_{1}}{\partial x^{3}}\right) \delta w_{1}+E I\left(\frac{\partial^{3} w_{-1}}{\partial x^{3}}\right) \delta w_{-1}+E I\left(\frac{\partial^{3} w_{0+}}{\partial x^{3}}\right) \delta w_{0}+E I\left(\frac{\partial^{3} w_{0-}}{\partial x^{3}}\right) \delta w_{0}$

The elementary work of the non-conservative forces is:

$$
\begin{aligned}
& \delta W_{n c}=E I\left(\frac{\partial^{2} w_{1}}{\partial x^{2}}\right) \delta\left(\frac{\partial w_{1}}{\partial x}\right)-E I\left(\frac{\partial^{2} w_{-1}}{\partial x^{2}}\right) \delta\left(\frac{\partial w_{-1}}{\partial x}\right) \\
& -E I\left(\frac{\partial^{3} w_{1}}{\partial x^{3}}\right) \delta w_{1}+E I\left(\frac{\partial^{3} w_{-1}}{\partial x^{3}}\right) \delta w_{-1}
\end{aligned}
$$

Because of a concentrated shear force created by LLR structure at $x=0$, it is $E I\left(\frac{\partial^{3} w_{0+}}{\partial x^{3}}\right) \neq E I\left(\frac{\partial^{3} w_{0-}}{\partial x^{3}}\right)$. Using the extended Hamilton's principle (25) with (51)(53) and separating the terms with $\delta w, \delta u_{1}$ and $\delta u_{2}$ the following equations are obtained:

$$
\begin{aligned}
& 0=-\rho A \frac{\partial^{2} w}{\partial t^{2}}-E I \frac{\partial^{4} w}{\partial x^{4}}+\left[k_{1}\left(u_{1}-w_{0}\right)-\frac{H}{D} k_{2}\left(u_{2}+\frac{H}{2 D}\left(w_{0}-u_{1}\right)\right)+\right. \\
& \left.E I\left(\frac{\partial^{3} w_{0+}}{\partial x^{3}}\right) \delta w_{0}+E I\left(\frac{\partial^{3} w_{0}-}{\partial x^{3}}\right) \delta w_{0}\right] \delta(x) \\
& -m_{1} \frac{\partial^{2} u_{1}}{\partial t^{2}}-k_{1}\left(u_{1}-w_{0}\right)+\frac{H}{D} k_{2}\left(u_{2}+\frac{H}{2 D}\left(w_{0}-u_{1}\right)\right)=0 \\
& m_{2} \frac{\partial^{2} u_{2}}{\partial t^{2}}+k_{2}\left(u_{2}+\frac{H}{2 D}\left(w_{0}-u_{1}\right)\right)=0
\end{aligned}
$$

Using the wave propagation function (46) and treating the system as a homogenized uniform metamaterial beam, the integration over the whole length gives: 


$$
\begin{aligned}
& 0=-\int_{-\frac{L}{2}}^{\frac{L}{2}} \rho A \frac{\partial^{2} w}{\partial t^{2}} d x-E I\left(\frac{\partial^{3} w_{1}}{\partial x^{3}}\right)+E I\left(\frac{\partial^{3} w_{-1}}{\partial x^{3}}\right)+k_{1}\left(u_{1}-w_{0}\right)-\frac{H}{D} k_{2}\left(u_{2}+\right. \\
& \left.\frac{H}{2 D}\left(w_{0}-u_{1}\right)\right) \\
& \widetilde{m} \frac{\partial^{2} w_{0}}{\partial t^{2}}+\tilde{k} w_{0}+k_{1}\left(u_{1}-w_{0}\right)-\frac{H}{D} k_{2}\left(u_{2}+\frac{H}{2 D}\left(w_{0}-u_{1}\right)\right)=0
\end{aligned}
$$

where:

$\tilde{m}=-\frac{2 \rho A \sin \left(\frac{\beta L}{2}\right)}{\beta}, \quad \tilde{k}=-2 E I \beta^{3} \sin \left(\frac{\beta L}{2}\right)$

Combining (58) with (55) and (56) we obtain:

$$
\left[\begin{array}{ccc}
-\widetilde{m} \omega^{2}+\tilde{k}-k_{1}-\frac{1}{2}\left(\frac{H}{D}\right)^{2} k_{2} & k_{1}+\frac{1}{2}\left(\frac{H}{D}\right)^{2} k_{2} & -\frac{H}{D} k_{2} \\
k_{1}+\frac{1}{2}\left(\frac{H}{D}\right)^{2} k_{2} & m_{1} \omega^{2}-k_{1}-\frac{1}{2}\left(\frac{H}{D}\right)^{2} k_{2} & \frac{H}{D} k_{2} \\
-\frac{H}{2 D} k_{2} & \frac{H}{2 D} k_{2} & m_{2} \omega^{2}-k_{2}
\end{array}\right] \times\left\{\begin{array}{l}
W_{0} \\
U_{1} \\
U_{2}
\end{array}\right\}=0
$$

To obtain the non-trivial solution, the determinant of the coefficient matrix should be set to 0 . If the solution of the determinant is a function of the wavenumber which is non-positive or even imaginary the wave propagation is stopped. So, the flexural waves are partially transformed into longitudinal waves and then totally blocked. It stimulates the lateral resonance to create inertial forces to counterbalance the shear forces resulting in wave suppression in the other directions. This type of beams is promising to be applied in the flexural absorber and isolator for vibration and noise control.

\section{Conclusions}

It can now be concluded:

1) The theory of a conventional vibration absorber (spring - mass system) is suitable for explanation of the basic concept of acoustic metamaterial with negative effective (dynamic) mass.

2) Acoustic metamaterial beams contain an isotropic beam with built-in springmass vibration absorbers. Depending on the way how the units are attached to the beam, the structure may absorb waves in one-direction (such as, for example, longitudinal waves) or waves in two directions (such as, for instance, transversal and longitudinal waves).

3) Usually, acoustic metamaterial beams may produce one or two frequency gaps. At the moment, it is of interest to produce beams for multi-frequency gaps. The stop-bands are rather small. They need to be enlarged.

4) We suggest introducing the construction of the acoustic metamaterial beams units which would have springs with nonlinear elastic properties instead of linear ones. Some investigation are already done (see [16-20]). The distinctive feature of this non-linear absorber would be that the vibration energy 
transferred to the absorber, would be either localized or dissipated internally and would not re-enter the main system, even if the excitation of the main system is halted.

\section{Acknowledgement}

The manuscript has been prepared with support of COST Action CA15125: Design for Noise Reducing Materials and Structures (DENORMS).

\section{References}

[1] E. P. Calius, X. Bremaud, B. Smith, A. Hall, Negative mass sound shielding structures: Early results, Physica Status Solidi B 246 (9), 2009. pp. 2089-2097

[2] H. Sun, X. Du, P. F. Pai, Theory of metamaterial beams for broadband vibration absorption, Journal of Intelligent Material Systems and Structures, 21, July, 2010, pp. 1085-1101

[3] P. F. Pai, H.Peng, S. Jiang, Acoustic metamaterial beams based on multifrequency vibration absorbers, International Journal of Mechanical Sciences 79, 2014, pp. 195-205

[4] V. G. Veselago, The electrodynamics of sustances with simultaneously negative values of $\varepsilon$ and $\mu$, Sov. Phys. Usp 10, 1968, pp. 509-514

[5] J. B. Pendry, Negative refraction makes a perfect lens, Phys. Rev. Lett. 85, 2000, pp. 3966-3969

[6] J. Valentine, S. Zhang, T. Zentgraf, E. Ulin-Avila, D. A. Genov, G. Bartal, $X$. Zhang, Three-dimensional optical metamaterial with a negative refractive index, Nature 455, 2008, pp. 376-379

[7] P. Sheng, X. X. Zhang, Z. Liu, C.T. Chan, Locally resonant sonic materials, Physica B 338, 2003, pp. 201-205

[8] G. W. Milton, J. R.Willis, On modifications of Newton's second law and linear continuum elastodynamics, Proceedings of the Royal Society A 463, 2007, pp. 855-880

[9] G. W. Milton, New metamaterials with macroscopic behavior outside that of continuum elastodynamics, New Journal of Physics 9, 359, 2007, pp. 113

[10] H. H. Huang, C. T. Sun, G. I Huang, On the negative effective mass density in acoustic metamaterials, International Journal of Engineering Science 47, 2009, pp. 610-617

[11] P. F. Pai, Metamaterial-based broadband elastic wave absorber, Journal of Intelligent Material Systems and Structures 21(5), 2010, pp. 517-528

[12] P. Sheng, X. X. Zhang, Z. Liu, C. T. Chan, Locally resonant sonic materials, Physica B 338, 2003, pp. 201-205 
[13] P. Sheng, J. Mei, Z. Liu, W. Wen, Dynamics mass density and acoustic metamaterials, Physica B 394, 2007, pp. 256-261

[14] R. Zhu, X. N. Liu, G. K. Hu, C. T. Sun, G. L. Huang, A chiral elastic metamaterial beam for broadband vibration suppression, Journal of Sound and Vibration 333, 2014, pp. 2759-2773

[15] T. Wang, M. P. Sheng, Q. H. Qin, Multi-flexural band gaps in an EulerBernoulli beam with lateral local resonators, Physics Letters A, 380, 2016, pp. 525-529

[16] P. F. Pai, B. Wen, A. S. Naser, M. J. Schulz, Structural vibration control using PZT patches and nonlinear phenomena, Journal of Sound and Vibration 215(2), 1998, pp. 273-296

[17] P. F. Pai, M. J. Schulz, A refined nonlinear vibration absorber, International Journal of Mechanical Sciences 42, 2000, pp. 537-560

[18] P. F. Pai, B. Rommel, M. J. Schulz, Nonlinear vibration absorbers using higher-order internal resonances, Journal of Sound and Vibration 234(5), 2000, pp. 799-817

[19] L. Cveticanin, M. Kalami-Yazdi, H. Askari, Analytical approximations to the solutions for a generalized oscillator with strong nonlinear terms, Journal of Engineering Mathematics 77(1), 2012, pp. 211-223

[20] M. M. Maaza, A. Arab, M. Belkhatir, S. Hammoudi, M. P. Luong, A. Benaissa, Non-linear behavior of sands under longitudinal resonance testing, Acta Polytechnica Hungarica 9(2), 2012, pp. 209-220

[21] L. Cveticanin, Gy. Mester, I. Biro, Parameter influence on the harmonically excited Duffing oscillator, Acta Polytechnica Hungarica 11(5), 2014, pp. $145-160$ 